\title{
Endovascular Therapy for Acute Stroke
}

\author{
Maria Politi (1), Andreas Kastrup (2), Kostas Marmagkiolis (3), Iris Grunwald (4), \\ Panagiotis Papanagiotou (1,4)
}

1. Clinic for Diagnostic and Interventional Neuroradiology, Hospital Bremen-Mitte

2. Clinic for Neurology, Hospital Bremen-Mitte

3. Pepin Heart Institute Florida Hospital, , Tampa FL; University of Missouri, Columbia MO

4. Neuroscience and Vascular Simulation Unit, Anglia Ruskin University, Essex, UK

Correspondence Author:

Panagiotis Papanagiotou

Clinic for Diagnostic and Interventional Neuroradiology,

Hospital Bremen-Mitte, Germany

St.-Jürgen Str. 1

Tel. 0049-421-4973625

e-mail: papanagiotou@me.com

\begin{abstract}
Stroke is the most common cause of permanent disability, the second most common cause of dementia, and the fourth most common cause of death in the Western world. Recently, based on positive multicenter randomized clinical trials, endovascular therapy (EVT) for acute stroke has undergone a revolution. Routine mechanical thrombectomy in addition to intravenous thrombolysis has been shown to provide excellent outcomes for patients with proximal anterior circulation occlusions. This procedure reduces disability and benefits are seen across a wide range of age and initial stroke severity. Important features that affect treatment decisions include time of presentation, the patient's clinical status, imaging characteristics, and lab tests. Under optimal conditions, it should be available to patients 24/7, similar to systems offering prompt percutaneous coronary interventions to patients with acute ST-segment elevation myocardial infarctions.
\end{abstract}




\section{Introduction}

Stroke is the most common cause of permanent disability, the second most common cause of dementia, and the fourth most common cause of death in the Western world ${ }^{1}$. The World Health Organization estimates that 5.7 million people die from stroke each year. Each year about 795,000 people in the United States and 1 million people in the European Union experience a new or recurrent stroke (ischemic or hemorrhagic). The majority (>80\%) of acute strokes are ischemic. The patients who survive are often burdened with exorbitant rehabilitation costs, lost wages and productivity, limitations in their daily social activity, and significant residual disability ${ }^{2}$. Given that the aging population of the world is increasing in size, the statistics of stroke incidence and prevalence are likely to also climb proportionately.

The drug therapy for acute ischemic stroke is thrombolysis with recombinant tissue plasminogen activator (t-PA), which has been shown in clinical trials to be effective ${ }^{3}$. However, among occlusions of large proximal vessels resulting in severe strokes, recanalization rates are reduced, resulting in an overall poor prognosis. For many years the concept of catheter directed delivery of thrombolytic medications was investigated. The hypothesis behind this treatment paradigm was that delivering thrombolytics directly to the site of arterial occlusion may provide faster vessel recanalization with fewer bleeding complications due to less thrombolytic drug use. The goal intra-arterial thrombolysis (IAT) was to decrease the level of disability caused by the stroke and perhaps increase the thrombolytic window beyond the 3 hours studied in the original NINDS trial with IV tPA ${ }^{4}$. The Prolyse in Acute Cerebral Thromboembolism II (PROACT II) randomized controlled study provided pilot data supporting this hypothesis ${ }^{5}$. However a recent meta-analysis comparing IAT to supportive medical therapy as well as to IV tPA failed to demonstrate compelling benefit ${ }^{6}$. 
Recently, based on positive multicenter randomized clinical trials, endovascular therapy (EVT) underwent a revolution ${ }^{7}$. Endovascular treatments increase the chance of successful and rapid recanalization. In recent years, much effort has been made to develop new revascularization devices that provide faster and higher recanalization rates in order to generate a better clinical outcome for patients ${ }^{8}$. Several randomized clinical trials have assessed this combined approach. Between December 2014 and August 2016, six randomized, controlled trials and individual patient data meta-analysis demonstrated that thrombectomy combined with standard medical treatment is more effective than standard medical treatment alone for severe acute stroke caused by large-vessel occlusion in anterior circulation ${ }^{7,9-14}$. International guidelines have been recently updated to include recommendation of EVT when possible $^{15}$.

\section{Background and Evidence}

\section{Recent randomized clinical trials}

Recently, the Multicenter Randomized Clinical Trial of Endovascular Treatment of Acute Ischemic Stroke in the Netherlands (MR CLEAN) demonstrated a clear overall benefit of adding EVT to standard care (including intravenous thrombolysis) in patients with acute ischemic stroke caused by proximal arterial occlusion of the anterior circulation, if administered within 6 hours from symptom onset ${ }^{7}$.

After publication of the results of MR CLEAN five subsequent trials ESCAPE (Endovascular Treatment for Small Core and Anterior Circulation Proximal Occlusion With Emphasis on Minimizing CT to Recanalization Times) ${ }^{13}$, EXTEND-IA (Extending the Time for Thrombolysis in Emergency Neurological Deficits - Intra-Arterial) ${ }^{11}$, SWIFT PRIME (Solitaire With the Intention for Thrombectomy as Primary Endovascular Treatment) ${ }^{12}$, REVASCAT (Endovascular Revascularization With Solitaire Device Versus Best Medical Therapy in Anterior Circulation Stroke Within 8 Hours After Symptom Onset in Ischemic 
Stroke) ${ }^{10}$, and THRACE (Mechanical thrombectomy after intravenous alteplase versus alteplase alone after stroke) ${ }^{14}$ demonstrated the efficacy of EVT versus IV-tPA alone in treating patients with acute anterior circulation ischemic stroke.

All six randomized, controlled trials, showed a benefit for endovascular treatment compared with IV t-PA alone regarding functional outcomes. The percentage of patients achieving a favorable clinical outcome with EVT varied between 33\% and 71\%; there was a consistent positive difference across all the studies with functional independence (defined as a modified Rankin Scale score of 0 to 2 at 90 days) between the interventional and control arms favoring EVT by $14 \%$ to $31 \%$ (Table 1). The difference between the groups was more pronounced in the trials in which penumbral imaging with CT perfusion was used. However, even without imaging selection beyond the unenhanced CT, such as in the MR CLEAN and THRACE studies, there was a clear benefit in favor of EVT.

In all studies the intra-cerebral hemorrhage risk in both the interventional and control arms ranged from $0 \%$ to $7 \%$. The fact that in all the trials EVT carried no higher bleeding risk or 90 days mortality rate compared with IV t-PA demonstrates that thrombectomy is safe and any bleeding risk is caused mainly by thrombolysis. There was an overall trend toward a reduction in mortality with EVT. Successful recanalization was defined as a Thrombolysis In Cerebral Infarction score of $2 \mathrm{~b}$ or 3 and varied between $59 \%$ and $88 \%$. The trials showed also that the likelihood of a good outcome increased with better recanalization.

\section{Meta-analysis}

A meta-analysis of individual patient data from the five first randomized trials addressed the fact that the degree of benefit conferred by endovascular thrombectomy is substantial: for every 100 patients treated, 38 will have a less disabled outcome than with best medical management and 20 more will achieve functional independence (mRS 0-2) as a result of treatment. The number needed to treat for one patient to have reduced disability of at least 1 point on mRS was $2.6^{9}$. 
For subgroup analysis of mRS distribution shift at 90 days, there was no evidence of heterogeneity of treatment eff ect across any of the pre-specified variables: age, gender, National Institutes of Health Stroke Scale (NIHSS) at admission, site of intracranial occlusion, intravenous alteplase received or ineligible, Alberta Stroke Program Early CT Score (ASPECTS), time from onset to randomization, and presence of tandem cervical carotid occlusion. The direction of eff ect favored endovascular treatment across all strata, although the adjusted ORs for treatment were not significant for patients younger than 50 years, those with a low ASPECTS or NIHSS score, or in those with an M2 segment thrombus. Eff ects favoring intervention were significant in several subgroups of special interest, including patients older than 80 years, those randomized more than 300 min after symptom onset, and those not receiving intravenous $\mathrm{t}-\mathrm{PA}^{9,16}$. There was also no evidence of heterogeneity of treatment eff ect across the pre-specified subgroups for achievement of functional independence (mRS 0-2) at 90 days.

The rates of symptomatic intracranial hemorrhage and radiological intracerebral hematoma are no higher with endovascular thrombectomy than with best medical therapy alone and mortality risk did not significantly differ between groups. No heterogeneity of treatment effect was noted for mortality ( $\mathrm{p}$ interaction $=0 \cdot 33$ ) but rate ratios were rarely significant in any of the subgroups. Patients older than 80 years assigned to thrombectomy had a slightly reduced risk of death assigned to control.

\section{Acute Stroke Therapy: Practical Aspects}

When treating acute stroke, time matters and optimized stroke protocols are necessary in order to avoid in-hospital delay and achieve the best possible outcome ${ }^{17}$. In practice less than one-third of the patients meet the goal of a "door-to-needle time" of $<60 \mathrm{~min}^{18}$. Therapeutic strategy should be designed, executed and monitored to emphasize expeditious assessment 
and treatment. An on-demand, 24-7-365, stroke reperfusion service is also required ${ }^{19,20}$. Important features that affect treatment decisions include time of presentation, the patient's clinical status, imaging characteristics, and lab tests.

\section{Time of presentation}

IV t-PA and EVT reperfusion therapies have both been shown to improve patient outcomes. However, the time window for treatment with either approach is limited. IV thrombolysis can be given up to $4.5 \mathrm{~h}$ after stroke onset; additional or primary EVT can be used up to $12 \mathrm{~h}$ after stroke onset. In anterior circulation strokes, the impact of successful thrombectomy is greater in the first 3 to $4.5 \mathrm{~h}$ after stroke compared with late recanalization after 5 to $8 \mathrm{~h}^{21}$. A metaanalysis of recent randomized trials showed that in patients who achieved substantial reperfusion with endovascular thrombectomy, each 1-hour delay to reperfusion was associated with a less favorable degree of disability and less functional independence, but no change in mortality ${ }^{22}$.

\section{Clinical examination}

A neurologic exam should be performed according to the National Institute of Health Stroke Scale (NIHSS). Precise and fast assessment of the NIHSS score requires some experience and should be practiced under non-acute circumstances. Patients with significant deficits manifesting scores between 8 and 20 are more likely to benefit from reperfusion with EVT, making them better candidates for treatment. Patients with minor to mild symptoms (NIHSS $<8)$ and an existing intracranial large vessel occlusion were not included in the trials. In these patients, the decision to perform additional EVT is based on the endovascular specialist's experience and the estimated risk of the procedure ${ }^{20}$.

\section{Imaging}

At the minimum a non-contrast CT scan should be performed to rule out intracerebral 
hemorrhage and stroke mimics (e.g., space occupying lesions) (Fig 1). ASPECTS quantitatively evaluates the core of ischemia on CT scans. ASPECTS is a topographic scoring system that divides the middle cerebral artery (MCA) territory of the brain affected by ischemic damage into 10 areas of interest. It is a strong predictor of both functional outcome and adverse events following treatment ${ }^{23}$. In a meta-analysis of the four studies that presented stratified ASPECTS data for mRS endovascular stroke treatment improved functional independence compared with best medical treatment in patients with high baseline ASPECTS $^{24}$. Mechanical thrombectomy also improved functional independence with moderate baseline ASPECTS. There was no evidence of benefit for endovascular stroke treatment in patients with low baseline ASPECTS. Overall, these results suggest that patients with baseline ASPECTS $>6$ benefit from endovascular stroke treatment. Clinically, an important distinction should be made between low ASPECTS as an indicator of a very poor prognosis and any possible treatment eff ect of reperfusion. If the prognosis is extremely poor, even a small treatment eff ect might not represent a useful intervention. ASPECTS is recommended by the American Society of Neuroradiology, American Heart Association/ American Stroke Association, European Stroke Organisation, and the Canadian Stroke Best Practice.

CT angiography confirms the existence of a large vessel occlusion, allows localization of the occluded vessel and may facilitate the intervention by obviating the need for cerebral angiography of non-target vessels (Fig 2). Moreover, it may identify collateral circulation and clot length ${ }^{25}$. A post hoc analysis of MR CLEAN neuroimaging data evaluated how the collateral status of the target vessel related to clinical outcome. Collaterals were graded on baseline CTA with a 4-point scale. This study demonstrated that baseline CTA collateral status modified the effect of EVT. The benefit of EVT was robust in patients with good collaterals on baseline CTA (Fig 2). Treatment benefit could not be established in patients with absent or poor collaterals ${ }^{26}$. However, in everyday clinical practice collateral status 
assessment on CTA can be prone to interobserver variability.

In two of the randomized trials (MR CLEAN and TRACE) imaging included mostly unenhanced CT scans and CT angiography.

Assessment of the presence and size of cerebral parenchymal damage guides candidacy for intravenous or intra-arterial therapy. If available MR- or CT-perfusion imaging can allow identification and quantification of the ischemic penumbra (ischemic, yet viable tissue at risk that may be salvaged by timely reperfusion), guiding further therapy, especially in wake-up strokes or presentations with onset of symptoms $>6$ hours or patients who are within 6 hours of symptom onset and have an ASPECTS $<6$.

Mobile CT scanners or flat panel CT in the catheterization laboratory and intensive care units may accelerate treatment decisions ${ }^{27}$.

\section{Lab tests}

Because time is critical, a limited number of essential diagnostic tests are recommended. A complete blood count, partial thromboplastin time (PTT), prothrombin time, serum creatinine, electrolytes, and glucose level should be obtained upon patient arrival in the emergency department. Only the assessment of blood glucose in addition to a head CT scan, must precede the initiation of IV rtPA. To shorten potential delays, portable point of care laboratory systems that allow measurement of these parameters at the site of imaging or in the emergency department have been shown to be useful ${ }^{17}$.

\section{Endovascular Procedure}

\section{Thrombectomy devices}

Based on the above recent positive multicenter randomized clinical trials, EVT has undergone a revolution. One of the main changes in these positive studies was the utilization of stent retriever devices. Stent retrievers are self-expandable stent-like devices that are fully retrievable. Therefore, these devices combine the advantages of prompt flow restoration and 
mechanical thrombectomy (Fig 3). The excellent recanalization results with low complication rates led to a high rate of favorable clinical outcome, confirming registry data and other randomized trials ${ }^{19,28,29}$.

However, clinical experience has reported situations that are resistant to stent retriever recanalization attempts. These situations include occlusions located in terminal internal carotid artery (tICA) and middle cerebral artery (MCA) bifurcation and trifurcation thrombi, as well as hard thrombi configuration. For these cases, direct aspiration of the thrombus can be used as an alternative technique. The aspiration technique has been an early component in the history of mechanical thrombectomy and has been published in a large number of small trials and clinical experiences ${ }^{30}$ (Fig 4). Over the course of time a great effort has been made to develop new aspiration devices, including changes in the distal inner diameters of the catheter. The main advantages of aspiration technique are the fast procedure time and the high rate of favorable clinical outcome if primary aspiration is successful. For direct thrombus aspiration, the most common aspiration catheters that are used are 5-F systems, e.g., the Penumbra ACE 64 reperfusion catheter (Penumbra Inc.), which has a 0.064-inch inner lumen and is significantly larger than the previous generation of aspiration catheters. Aspiration is performed with a pump that is capable of maintaining a nearly pure vacuum of $29 \mathrm{inHg}$ (98.2 $\mathrm{kPa}$ ). An aspiration catheter with a 0.068" distal inner diameter is already available (ACE 68, Penumbra Inc.) for clinical use ${ }^{28}$.

\section{General anesthesia vs. conscious sedation}

Endovascular thrombectomy is performed under general anesthesia with intubation or under conscious sedation. Some interventionalists and stroke physicians prefer general anesthesia with intubation, assuming it may be associated with less pain, anxiety, agitation, and movement, and lower aspiration risk. Others favor conscious sedation to save time, evoke less hemodynamic instability, and risk fewer ventilation-associated complications (sedation). 
Pathophysiological considerations concerning general anesthesia is anesthesia-associated hypotension and hypocapnia, the latter potentially causing cerebral vasoconstriction influencing the collateral status.

MR CLEAN investigators have reported that the outcomes of the 79 patients in the endovascular group who received general anesthesia were no different from the 267 nonendovascular control patients, whereas for the 137 endovascular patients who did not receive general anesthesia the outcomes were better than for the 267 control patients ${ }^{31}$. Similar data showing worse outcomes in those undergoing general anesthesia as compared to conscious sedation for endovascular were reported in a recent meta-analysis of 9 non-randomized studies. However, all these studies have selection bias, since general anesthesia was often chosen for patients with more severe illness ${ }^{32}$.

In contrast, in a single-center trial published recently, 150 randomized patients with acute ischemic stroke were to receive either general anesthesia with intubation or conscious sedation without intubation during thrombectomy. Early improvement in neurological function was not significantly different between treatment groups. Functional independence at 90 days, as reflected by a modified Rankin Scale score of 0 to 2, was significantly more frequent in the general anesthesia group (37\%) than in the conscious sedation group (18.2\%) 33. Notable in this study is the low rate of favorable clinical outcome at 90 days with only $18.2 \%$ in the conscious sedation group. Another critical issue of the study was that both general anesthesia and conscious sedation were performed by neurologists and not anesthesiologists.

Thus, a lack of clear superiority data between general anesthesia or conscious sedation suggests that interventionalists should choose the preferred method based on experience pending further studies.

\section{Technique}


With rare exceptions, femoral access is obtained. The radial artery may also be used in cases where puncture of the femoral arteries is not possible. To perform an intracranial thrombectomy it is necessary to place the long 6-F sheath or the 8-F guide catheter in the internal carotid artery (ICA). To place the sheath in the ICA, it is necessary to use a coaxial 125-cm long catheter over a guidewire. Two catheters can be used, the multipurpose catheter for a type I aortic arch configuration and the SIM2 catheter for type II and III aortic arch configurations.

After placement of the long 6-F sheath in the ICA, if possible in the petrous part, the occlusion of the target vessel is verified angiographically. The target vessel is entered with a 0.014-inch guidewire and a suitable microcatheter between 0.018 and 0.027 inches. The thrombus is crossed with the guidewire, and the microcatheter is placed distal to the thrombus. The stent retriever device is advanced to the distal end of the microcatheter. Then the microcatheter is removed to deploy the device under fluoroscopy. The device should extend beyond the proximal and distal edges of the thrombus. A control angiogram is performed after successful unfolding of the device. The sizes of stent retriever devices range from $3.0 x 15 \mathrm{~mm}$ to $6.0 \mathrm{x} 40 \mathrm{~mm}$. Stent retriever device selection depends on the size of the occluded artery (Fig 3). Typically, a 4- to 4.5-mm device is used for the middle cerebral artery (MCA) and a 6.0-mm device for occlusions in the terminal ICA. After a short period of time, the device is retrieved with continuous aspiration. If a balloon catheter is used, the balloon is inflated before retrieving the thrombectomy device. The procedure is repeated until a Thrombolysis In Cerebral Infarction score of $2 b$ or 3 is reached ${ }^{19}$.

If aspiration technique is used, the thrombus is crossed with the microwire and microcatheter, and the aspiration catheter is placed directly in the proximal part of the thrombus. The microwire and the microcatheter are removed. Entrapment of the thrombus is indicated by the absence of backflow. The catheter is then retrieved with constant negative pressure to avoid loss of thrombus. After each retrieval of clot fragments, the procedure is 
repeated until a TICI score of $\geq 2$ or 3 was reached (Fig 4).

Another technique is a combination of the two reperfusion systems, deploying the stent retriever in the thrombus, then placing the aspiration catheter at the proximal end of the stent retriever and pulling back both of them simultaneously under continuous aspiration.

\section{Everyday clinical practice}

The patients included in the randomized trials were carefully selected and the results were based on a very efficient workflow. Therefore, an interesting question arises: Can the promising results of these trials also be achieved in everyday clinical practice? A prospectively obtained database of a high-volume stroke center compared the early clinical outcome in patients with anterior circulation large vessel occlusions treated either with systemic thrombolysis or EVT with stent retrievers. The analysis in a total of 779 patients showed that significantly more patients in the endovascular treatment group than in the intravenous thrombolysis group had a favorable early clinical outcome (mRS 0-2 at discharge $37 \%$ vs. $27 \%, \mathrm{p}<0.01$ ) in everyday clinical practice. Similarly, significantly more patients in the EVT group than in the IVT group had an excellent early clinical outcome (mRS 0-1) (20\% vs. $13 \%, \mathrm{p}<0.01)$. The infarct sizes were significantly smaller after endovascular treatment than after intravenous thrombolysis, whereas the symptomatic intracranial hemorrhages rate and in-hospital mortality were comparable between both treatment groups ${ }^{34}$.

\section{Costs}

Stroke itself is an expensive disease in terms of its societal, personal, and financial impact. Rationalization concerning the clinical value and effectiveness of thrombectomy is guided by information regarding the benefits, risks, and associated costs. Recent cost-effectiveness analysis based on the UK National Health Service shows that although the upfront costs of thrombectomy are high, the potential reduction in morbidity can result in savings downstream both in the hospital and in the community setting, resulting in a significant reduction in the 
overall economic burden of stroke $\mathrm{e}^{35}$.

\section{Conclusion}

Routine endovascular thrombectomy has been shown to provide excellent outcomes for patients with proximal anterior circulation occlusions. This procedure reduces disability and benefits are seen across a wide range of age and initial stroke severity. Under optimal conditions, this treatment should be available to stroke patients at most locations 24/7, similar primary percutaneous coronary interventions for patients with acute ST-segment elevation myocardial infarctions. To accomplish this, a sufficient number of centers with acute stroke management systems in place and an adequate number of well-trained physicians capable of performing EVT for stroke are needed.

\section{References}

1. Rothwell PM, Coull AJ, Silver LE, et al. Population-based study of event-rate, incidence, case fatality, and mortality for all acute vascular events in all arterial territories (Oxford Vascular Study). Lancet. 2005;366(9499):1773-1783. doi:10.1016/S0140-6736(05)67702-1.

2. Writing Group Members, Mozaffarian D, Benjamin EJ, et al. Heart Disease and Stroke Statistics-2016 Update: A Report From the American Heart Association. Circulation. 2016;133(4):e38-e360. doi:10.1161/CIR.0000000000000350.

3. Hacke W, Kaste M, Bluhmki E, et al. Thrombolysis with alteplase 3 to 4.5 hours after acute ischemic stroke. $N$ Engl J Med. 2008;359(13):1317-1329. doi:10.1056/NEJMoa0804656.

4. Kwiatkowski TG, Libman RB, Frankel M, et al. Effects of tissue plasminogen activator for acute ischemic stroke at one year. National Institute of Neurological Disorders and Stroke Recombinant Tissue Plasminogen Activator Stroke Study Group. N Engl J Med. 1999;340(23):1781-1787. doi:10.1056/NEJM199906103402302.

5. Furlan A, Higashida R, Wechsler L, et al. Intra-arterial prourokinase for acute ischemic stroke. The PROACT II study: a randomized controlled trial. Prolyse in Acute Cerebral Thromboembolism. JAMA. 1999;282(21):2003-2011.

6. Nam J, Jing H, O'Reilly D. Intra-arterial thrombolysis vs. standard treatment or intravenous thrombolysis in adults with acute ischemic stroke: a systematic review and meta-analysis. Int J Stroke. 2015;10(1):13-22. doi:10.1111/j.1747-4949.2012.00914.x.

7. Berkhemer OA, Fransen PSS, Beumer D, et al. A randomized trial of intraarterial 
treatment for acute ischemic stroke. N Engl J Med. 2015;372(1):11-20.

doi:10.1056/NEJMoa1411587.

8. Papanagiotou P, Roth C, Walter S, et al. Treatment of acute cerebral artery occlusion with a fully recoverable intracranial stent: a new technique. Circulation.

2010;121(23):2605-2606. doi:10.1161/CIRCULATIONAHA.110.948166.

9. Goyal M, Menon BK, van Zwam WH, et al. Endovascular thrombectomy after largevessel ischaemic stroke: a meta-analysis of individual patient data from five randomised trials. Lancet. 2016;387(10029):1723-1731. doi:10.1016/S01406736(16)00163-X.

10. Jovin TG, Chamorro Á, Cobo E, et al. Thrombectomy within 8 hours after symptom onset in ischemic stroke. $N$ Engl J Med. 2015;372(24):2296-2306.

doi:10.1056/NEJMoa1503780.

11. Campbell BCV, Mitchell PJ, Kleinig TJ, et al. Endovascular therapy for ischemic stroke with perfusion-imaging selection. N Engl J Med. 2015;372(11):1009-1018. doi:10.1056/NEJMoa1414792.

12. Saver JL, Goyal M, Bonafe A, et al. Stent-retriever thrombectomy after intravenous tPA vs. t-PA alone in stroke. $N$ Engl J Med. 2015;372(24):2285-2295. doi:10.1056/NEJMoa1415061.

13. Goyal M, Demchuk AM, Menon BK, et al. Randomized assessment of rapid endovascular treatment of ischemic stroke. $N$ Engl J Med. 2015;372(11):1019-1030. doi:10.1056/NEJMoa1414905.

14. Bracard S, Ducrocq X, Mas JL, et al. Mechanical thrombectomy after intravenous alteplase versus alteplase alone after stroke (THRACE): a randomised controlled trial. Lancet Neurol. 2016;15(11):1138-1147. doi:10.1016/S1474-4422(16)30177-6.

15. Powers WJ, Derdeyn CP, Biller J, et al. 2015 American Heart Association/American Stroke Association Focused Update of the 2013 Guidelines for the Early Management of Patients With Acute Ischemic Stroke Regarding Endovascular Treatment: A Guideline for Healthcare Professionals From the American Heart Association/American Stroke Association. Stroke. 2015;46(10):3020-3035. doi:10.1161/STR.0000000000000074.

16. Marmagkiolis K, Hakeem A, Cilingiroglu M, et al. Safety and Efficacy of Stent Retrievers for the Management of Acute Ischemic Stroke: Comprehensive Review and Meta-Analysis. JACC Cardiovasc Interv. 2015;8(13):1758-1765. doi:10.1016/j.jcin.2015.07.021.

17. Walter S, Kostopoulos P, Haass A, et al. Point-of-care laboratory halves door-totherapy-decision time in acute stroke. Ann Neurol. 2011;69(3):581-586. doi:10.1002/ana.22355.

18. White CJ. Don“t hurt my brain ... it”s my second favorite organ. JACC CardiovasC Interv. 2013;6(4):392-393. doi:10.1016/j.jcin.2013.01.133.

19. Papanagiotou P, Roth C, Walter S, et al. Carotid artery stenting in acute stroke. $J$ Am Coll Cardiol. 2011;58(23):2363-2369. doi:10.1016/j.jacc.2011.08.044. 
20. Papanagiotou P, White CJ. Endovascular Reperfusion Strategies for Acute Stroke. JACC Cardiovasc Interv. 2016;9(4):307-317. doi:10.1016/j.jcin.2015.11.014.

21. Nogueira RG, Liebeskind DS, Sung G, et al. Predictors of good clinical outcomes, mortality, and successful revascularization in patients with acute ischemic stroke undergoing thrombectomy: pooled analysis of the Mechanical Embolus Removal in Cerebral Ischemia (MERCI) and Multi MERCI Trials. Stroke. 2009;40(12):3777-3783. doi:10.1161/STROKEAHA.109.561431.

22. Saver JL, Goyal M, van der Lugt A, et al. Time to Treatment With Endovascular Thrombectomy and Outcomes From Ischemic Stroke: A Meta-analysis. JAMA. 2016;316(12):1279-1288. doi:10.1001/jama.2016.13647.

23. Nagel S, Sinha D, Day D, et al. e-ASPECTS software is non-inferior to neuroradiologists in applying the ASPECT score to computed tomography scans of acute ischemic stroke patients. Int J Stroke. 2016;355:174749301668102. doi:10.1177/1747493016681020.

24. Balami JS, Sutherland BA, Edmunds LD, et al. A systematic review and meta-analysis of randomized controlled trials of endovascular thrombectomy compared with best medical treatment for acute ischemic stroke. Int J Stroke. 2015;10(8):1168-1178. doi:10.1111/ijs.12618.

25. Wasser K, Papanagiotou P, Brunner F, et al. Impact of ASPECTS on computed tomography angiography source images on outcome after thrombolysis or endovascular therapy in large vessel occlusions. Eur J Neurol. 2016;23(11):1599-1605. doi:10.1111/ene.13068.

26. Berkhemer OA, Jansen IGH, Beumer D, et al. Collateral Status on Baseline Computed Tomographic Angiography and Intra-Arterial Treatment Effect in Patients With Proximal Anterior Circulation Stroke. Stroke. 2016;47(3):768-776. doi:10.1161/STROKEAHA.115.011788.

27. Walter S, Kostopoulos P, Haass A, et al. Diagnosis and treatment of patients with stroke in a mobile stroke unit versus in hospital: a randomised controlled trial. Lancet Neurol. 2012;11(5):397-404. doi:10.1016/S1474-4422(12)70057-1.

28. Roth C, Papanagiotou P, Behnke S, et al. Stent-assisted mechanical recanalization for treatment of acute intracerebral artery occlusions. Stroke. 2010;41(11):2559-2567. doi:10.1161/STROKEAHA.110.592071.

29. Roth C, Reith W, Walter S, et al. Mechanical recanalization with flow restoration in acute ischemic stroke: the ReFlow (mechanical recanalization with flow restoration in acute ischemic stroke) study. JACC Cardiovasc Interv. 2013;6(4):386-391. doi:10.1016/j.jcin.2012.11.013.

30. Grunwald IQ, Walter S, Papanagiotou P, et al. Revascularization in acute ischaemic stroke using the penumbra system: the first single center experience. Eur J Neurol. 2009;16(11):1210-1216. doi:10.1111/j.1468-1331.2009.02750.x.

31. Berkhemer OA, van den Berg LA, Fransen PSS, et al. The effect of anesthetic management during intra-arterial therapy for acute stroke in MR CLEAN. Neurology. 2016;87(7):656-664. doi:10.1212/WNL.0000000000002976. 
32. Ouyang F, Chen Y, Zhao Y, Dang G, Liang J, Zeng J. Selection of Patients and Anesthetic Types for Endovascular Treatment in Acute Ischemic Stroke: A MetaAnalysis of Randomized Controlled Trials. Ai J, ed. PLoS ONE. 2016;11(3):e0151210. doi:10.1371/journal.pone.0151210.

33. Schönenberger S, Uhlmann L, Hacke W, et al. Effect of Conscious Sedation vs General Anesthesia on Early Neurological Improvement Among Patients With Ischemic Stroke Undergoing Endovascular Thrombectomy: A Randomized Clinical Trial. JAMA. 2016;316(19):1986-1996. doi:10.1001/jama.2016.16623.

34. Kastrup A, Brunner F, Wasser K, et al. Endovascular therapy versus thrombolysis in patients with anterior circulation stroke in everyday clinical practice. Int J Stroke. 2016;11(5):544-548. doi:10.1177/1747493016641948.

35. Lobotesis K, Veltkamp R, Carpenter IH, Claxton LM, Saver JL, Hodgson R. Costeffectiveness of stent-retriever thrombectomy in combination with IV t-PA compared with IV t-PA alone for acute ischemic stroke in the UK. J Med Econ. 2016;19(8):785794. doi:10.1080/13696998.2016.1174868. 